\title{
Dye-Assisted Gain of Strongly Confined Surface Plasmon Polaritons in Silver Nanowires
}

Aniruddha Paul, ${ }^{\dagger}$ Yu-Rong Zhen,,${ }^{\dagger}$ Yi Wang, ${ }^{\S}$ Wei-Shun Chang, ${ }^{\dagger}$ Younan Xia, ${ }^{\S}$ Peter Nordlander ${ }^{\ddagger}, \|$ and Stephan Link ${ }^{\dagger, \|, *}$

†Department of Chemistry, ${ }^{\dagger}$ Department of Physics and Astronomy, ${ }^{\|}$Department of Electrical and Computer Engineering, Laboratory for Nanophotonics, Rice University, Houston, Texas 77005, United States.

${ }^{\S}$ The Wallace H. Coulter Department of Biomedical Engineering, Georgia Institute of Technology and Emory University, Atlanta, Georgia 30332, United States.

*Correspondence: slink@rice.edu 
Subwavelength confinement and active control of light is essential for nanoscale communication devices at visible frequencies that support large bandwidths. $^{1-5}$ Noble-metal nanostructures present an excellent platform for strongly confined optical waveguides ${ }^{6-13}$ because of their ability to support surface plasmon polaritons (SPPs). ${ }^{14}$ However, SPP propagation suffers from losses that seriously limit their application potential. ${ }^{9}$ Although significant progress toward SPP loss compensation has been reported for various planar 2D waveguide structures, ${ }^{15-20}$ as well as lasing involving strongly localized plasmon modes, ${ }^{21,22}$ SPP gain in 1D nanoscale waveguides at visible frequencies is yet to be accomplished. Here, we report the first demonstration of gain for deep subwavelength confined SPPs $\left(\operatorname{mode}\right.$ area $\left.=\lambda^{2} / 40\right)$ in chemically prepared silver nanowires (Ag NWs). We measured a gain coefficient of $270 \mathrm{~cm}^{-1}$ resulting in $14 \%$ loss compensation using a continuous-wave (cw) pump laser. These results are an important step toward total loss compensation for highly confined nanowire SPPs.

Considerable efforts in achieving SPP loss compensation have led to the realization of SPP gain through stimulated emission of weakly confined SPPs supported on metal film and strip waveguides. ${ }^{15-18,23-25}$ Optical gain has been demonstrated by measuring amplified spontaneous emission for a thin film of gold covered with a fluorescent polymer. ${ }^{18}$ Enhancement in the SPP propagation length and partial loss compensation has been achieved for SPPs supported at the interface of a quantum dotdoped polymer strip on a gold film. ${ }^{17}$ Complete loss compensation and net output amplification of SPPs in strip waveguides of gold have been realized via an optical gain medium by De Leon and Berini, ${ }^{19}$ and more recently by Kena-Cohen et al. ${ }^{20}$ However, to 
date there exists no demonstration of optical gain for subwavelength-confined SPPs like those in metallic NWs, despite the fact that plasmon waveguiding in 1D silver and gold NWs is well documented. ${ }^{8-13,26-28}$

Chemically synthesized metal NWs with lateral dimensions of $\sim 100 \mathrm{~nm}$ have emerged as an important class of plasmonic waveguides ${ }^{8-11,27,28}$ supporting strongly confined SPPs. SPP propagation lengths in NWs are significantly enhanced compared to polycrystalline waveguides prepared lithographically because of reduced defect scattering and leakage radiation. ${ }^{9,10}$ Apart from their waveguiding properties, studied extensively by near-field ${ }^{10,13}$ and fluorescence-based imaging techniques, ${ }^{26-28}$ coupled NW structures have been shown to exhibit device functionalities such as nanoscale routers, modulators and logic elements. ${ }^{12,29}$ Despite reduced radiative losses, SPPs in chemically prepared NWs still suffer from absorptive losses (on the order of $-0.4 \mathrm{~dB}_{\mu \mathrm{m}}^{-1}$ at $800 \mathrm{~nm}$ ) because of strong confinement. ${ }^{27}$ These losses represent an impediment toward the realization of integrated plasmonic circuitry. In this study, we demonstrate for the first time SPP gain in chemically prepared Ag NWs that support SPPs with a mode area of only $\lambda^{2} / 40$.

SPP gain measurements were performed on individual Ag NWs by optically pumping a gain medium consisting of a poly(methyl methacrylate) (PMMA) film containing Rhodamine 640 (R640), as schematically illustrated in Figure 1. SPPs were launched by exciting one NW end with a $633 \mathrm{~nm}$ cw probe laser. The optical output due to conversion of SPPs into photons from the opposite (output) end of the NW was monitored (Figure 1A). Then, the measurement was repeated with optical pumping of the dye molecules with $514 \mathrm{~nm}$ cw laser light focused in the middle of the NW (Figure 1B). 
SPP gain was analyzed by comparing the output signal intensities with and without pumping the dye-doped polymer film.

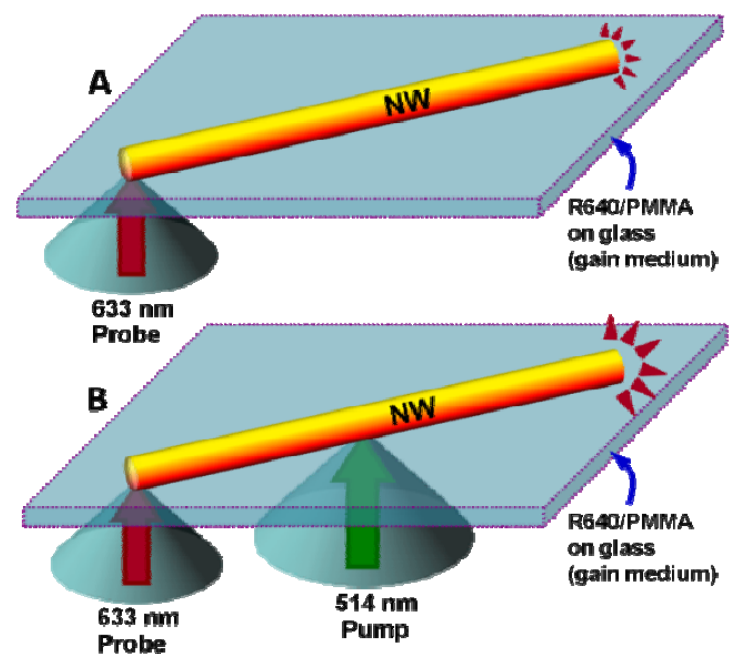

Figure 1. Schematic description of the SPP gain experiment: (A) The SPPs in an Ag NW, placed on top of a gain medium consisting of a dye-doped polymer (R640 in PMMA), were excited by a $633 \mathrm{~nm}$ probe laser at the left end and the output from the opposite end was monitored. (B) A second $514 \mathrm{~nm}$ pump laser was focused in the middle of the NW that optically pumped the gain medium below the NW, resulting in a significant enhancement of the output signal at the right end. See Methods and Supporting Information sections for experimental details.

Experimental manifestation of SPP gain is illustrated in Figure 2, which summarizes the results for a representative NW. Figure 2A shows a CCD camera image of a single Ag NW recorded under $633 \mathrm{~nm}$ excitation at the left end of the NW with a laser power of $12 \mathrm{~W} / \mathrm{cm}^{2}$. Direct conversion between photons and SPPs is only possible at the ends of a NW where symmetry breaking overcomes the momentum mismatch. ${ }^{10}$ Figure 2B shows the same NW illuminated only in its middle section with the $514 \mathrm{~nm}$ pump laser at a power of $400 \mathrm{~W} / \mathrm{cm}^{2}$. In addition to intense fluorescence emitted from the pump region, both ends of the NW emit due to coupling of the spontaneous emission of the dye molecules into SPP modes. The image in Figure 2C shows the same NW with both probe and pump excitation. Optical gain was achieved if the output signal at the 
right NW end in Figure 2C (pump+probe) was enhanced compared to the value obtained from Figure 2A (probe only) after correcting for the background spontaneous emission in Figure 2B (pump only).
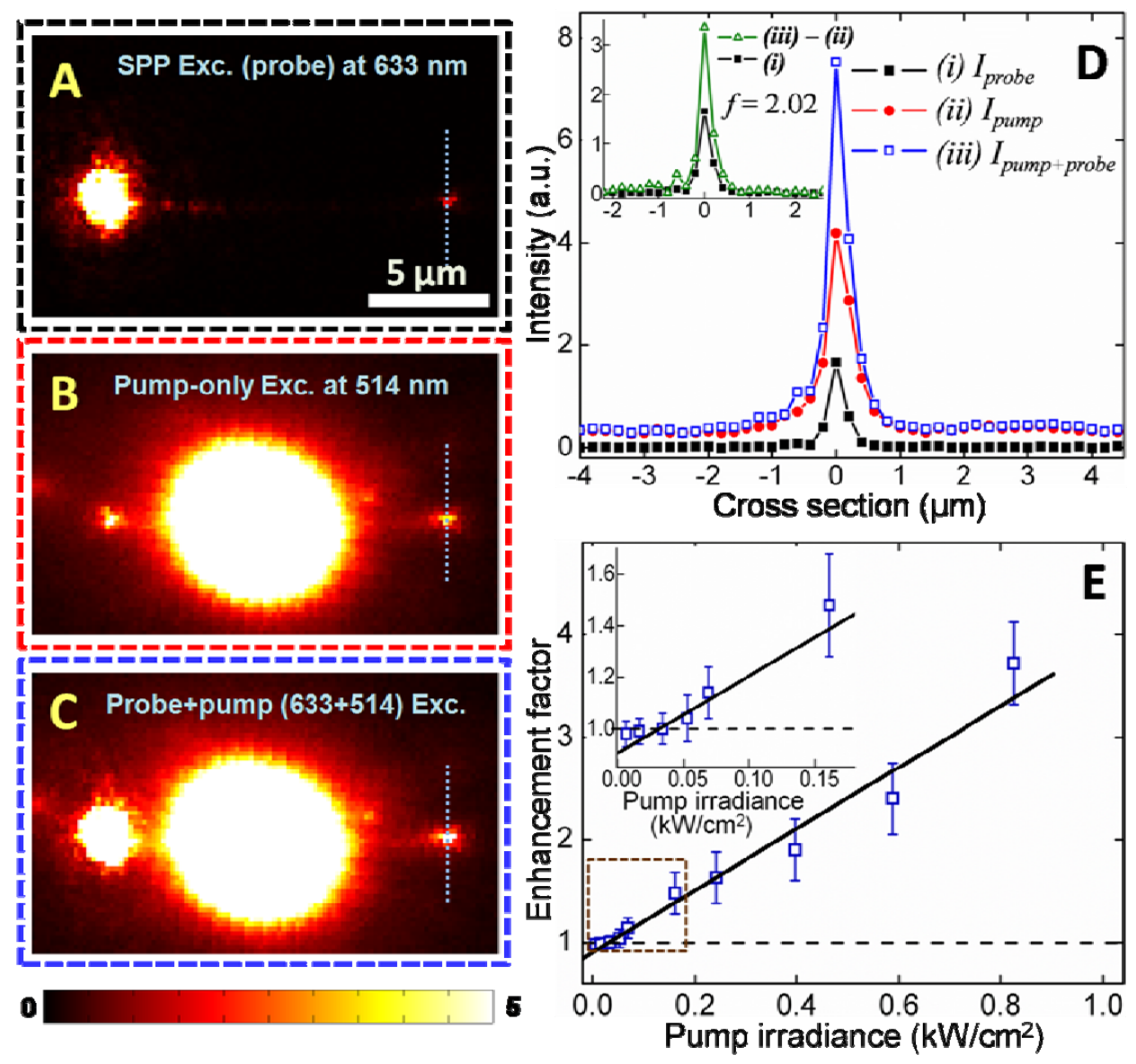

Figure 2. Experimental realization of SPP gain: (A) CCD camera image of an Ag NW excited at the left end by a $633 \mathrm{~nm} \mathrm{cw}$ probe laser $\left(12 \mathrm{~W} / \mathrm{cm}^{2}\right)$, which generated SPPs that were converted back into photons at the opposite NW end. (B) Optical image of the same NW, which was excited with a cw $514 \mathrm{~nm}$ pump laser $\left(400 \mathrm{~W} / \mathrm{cm}^{2}\right)$ in its middle section. The optical output from both NW ends was due to spontaneous dye fluorescence, which was coupled into the NW waveguide and then emitted at both NW ends. (C) Optical image of the same NW with combined excitation of both $633 \mathrm{~nm}$ probe and $514 \mathrm{~nm}$ pump lasers, keeping the laser powers and integration time the same as before. (D) Intensity line sections of the output signal, as indicated by the dashed lines in $\mathrm{A}-\mathrm{C}$, for the three respective images: $I_{\text {probe }}, I_{\text {pump }}$ and $I_{\text {pump }+ \text { probe }}$ corresponding to probe only (i), pump only (ii), and combined pump+probe (iii) excitation, respectively. The inset shows that after subtraction of the pump only signal the output intensity for the combined pump+probe excitation was larger than if only the probe was on, i.e. $I_{\text {pump }+ \text { probe }}-I_{\text {pump }}>I_{\text {probe }}$. This enhancement of the output signal when the pump was on indicates SPP gain. (E) Pump irradiance dependence of the output signal enhancement factor, $f$, and linear fit for values above the threshold for SPP gain, i.e. $f>1$. The inset shows a magnified view for low pump irradiances (dotted box). 
A quantitative analysis of the data in Figures 2A-C demonstrates a 2-fold enhancement of the optical output signal at a pump power of $400 \mathrm{~W} / \mathrm{cm}^{2}$ (Figures 2D and 2E). Intensity line sections of the output signal perpendicular to the NW as indicated by the dashed lines in the optical images are shown in Figure 2D for the three excitation conditions. The inset demonstrates clear enhancement of the output signal with optical pumping, i.e. $I_{\text {pump }+ \text { probe }}-I_{\text {pump }}>I_{\text {probe }}$. To quantify gain, we define the output signal enhancement factor:

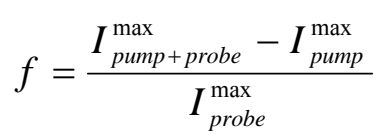

where the superscript $I^{\max }$ refers to the peak value of the corresponding intensity line sections in Figure 2D and the gain threshold was reached when $f>1$.

A clear threshold for SPP gain was observed as a function of pump irradiance (Figure 2E). The value of $f$ at each pump power was obtained by averaging the results of 7 NWs. An output signal enhancement factor of $f>1$ was evident at a pump irradiance of $50 \mathrm{~W} / \mathrm{cm}^{2}$ (inset of Figure 2E). Above this threshold $f$ increased linearly with pump irradiance. The observation of both threshold and linear pump power dependence are signatures of stimulated SPP emission. ${ }^{16,19}$ The maximum output signal enhancement achieved was $f=3.65$ at a pump irradiance of $800 \mathrm{~W} / \mathrm{cm}^{2}$. For larger pump powers, photobleaching of the dye molecules became too severe to achieve reliable results (Supporting Information).

Measuring the spectral characteristics of the optical output without and with optical pumping provided further insight into the stimulated emission of SPPs and optical gain in single Ag NWs (Figure 3A). Emission spectra at the NW end were acquired using 
a grating spectrograph. The desired area was selected with a slit to avoid stray light contamination. The spectrum with probe-only excitation (i) shows the sharp characteristic
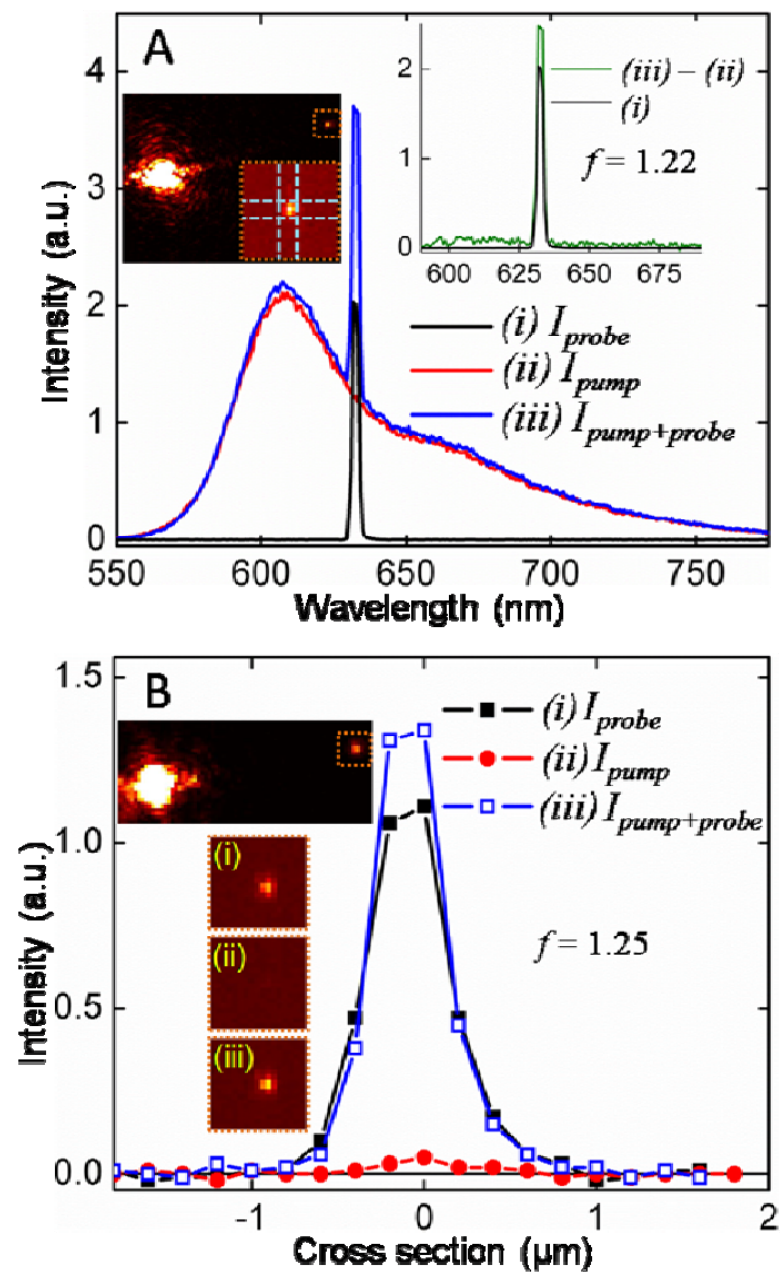

Figure 3. Spectral characteristics of the output signal enhancement. (A) Spectra collected with an integration time of 0.3 seconds from the light emitted at the output end of an Ag NW under the three different excitation conditions discussed in Figure 2: (i) $633 \mathrm{~nm}$ probe only $\left(12 \mathrm{~W} / \mathrm{cm}^{2}\right)$; (ii) $514 \mathrm{~nm}$ pump only $\left(400 \mathrm{~W} / \mathrm{cm}^{2}\right)$; (iii) pump+probe. The left inset shows an optical image of the NW investigated and highlights the area (dotted square) from which the output signal was collected (see Methods). The right inset shows that an output signal enhancement factor of $f=$ 1.22 was obtained from the spectra at the probe wavelength of $633 \mathrm{~nm}$. (B) SPP gain experiment carried out in image mode but with a $633 \pm 0.5 \mathrm{~nm}$ bandpass filter inserted in the detection path. Optical images are given as insets showing the Ag NW as well as magnified views of only the output end for (i) probe-only, (ii) pump-only, and (iii) combined pump+probe excitations, with the corresponding intensity line sections $I_{\text {probe }}, I_{\text {pump }}$, and $I_{\text {pump }+ \text { probe }}$ given in the main part of panel B. Probe and pump powers and integration time were the same as before. An output signal enhancement factor of $f=1.25$ was calculated in this case in excellent agreement with the spectral SPP gain measurements in panel A. Compared to the results in Figure 2 at the same pump power $(f=2.02)$, the smaller value for $f$ here means that wavelengths other than the $633 \mathrm{~nm}$ probe were also enhanced. 
laser line peaked at $633 \mathrm{~nm}$, whereas the spectrum for pump-only excitation (ii) represents the spontaneous emission of R640 (Figure S1), consistent with energy transfer from the excited dye molecules to SPPs. Under pump+probe excitation (iii), the spectrum consisted of both features. However, as in the image mode measurement (Figure 2), the overall intensity of this spectrum was greater than the sum of the two spectra (i) and (ii). Specifically, an output enhancement of $f=1.22$ at the $633 \mathrm{~nm}$ probe wavelength was calculated when the spectrum of pump-only excitation was subtracted from that of pump+probe excitation, and the difference was compared to the spectrum of probe-only excitation (inset in Figure 3A).

To further verify the spectral measurements, we also determined the output signal enhancement in the image mode configuration by adding a $633 \pm 0.5 \mathrm{~nm}$ bandpass filter in front of the detector and a pinhole in the first microscope image plane to spatially select only light from the NW output end (Figure 3B). For $400 \mathrm{~W} / \mathrm{cm}^{2}$ pump power the enhancement factor was $f=1.25$, in excellent agreement with the spectral measurements described in Figure 3A. At the highest possible pump power of $800 \mathrm{~W} / \mathrm{cm}^{2}$ the enhancement factor increased to $f=1.50$.

The enhancement factor at $633 \mathrm{~nm}$ was smaller than the overall enhancement factors measured at the same pump power in image mode (Figure 2D) and from integrating the three spectra in Figure $3 \mathrm{~A}$ over all wavelengths. This result shows that a significant portion of the overall signal enhancement originated from amplification of spontaneous background emission over the entire wavelength range of the dye fluorescence and not just at the $633 \mathrm{~nm}$ probe wavelength. This broadband enhancement 

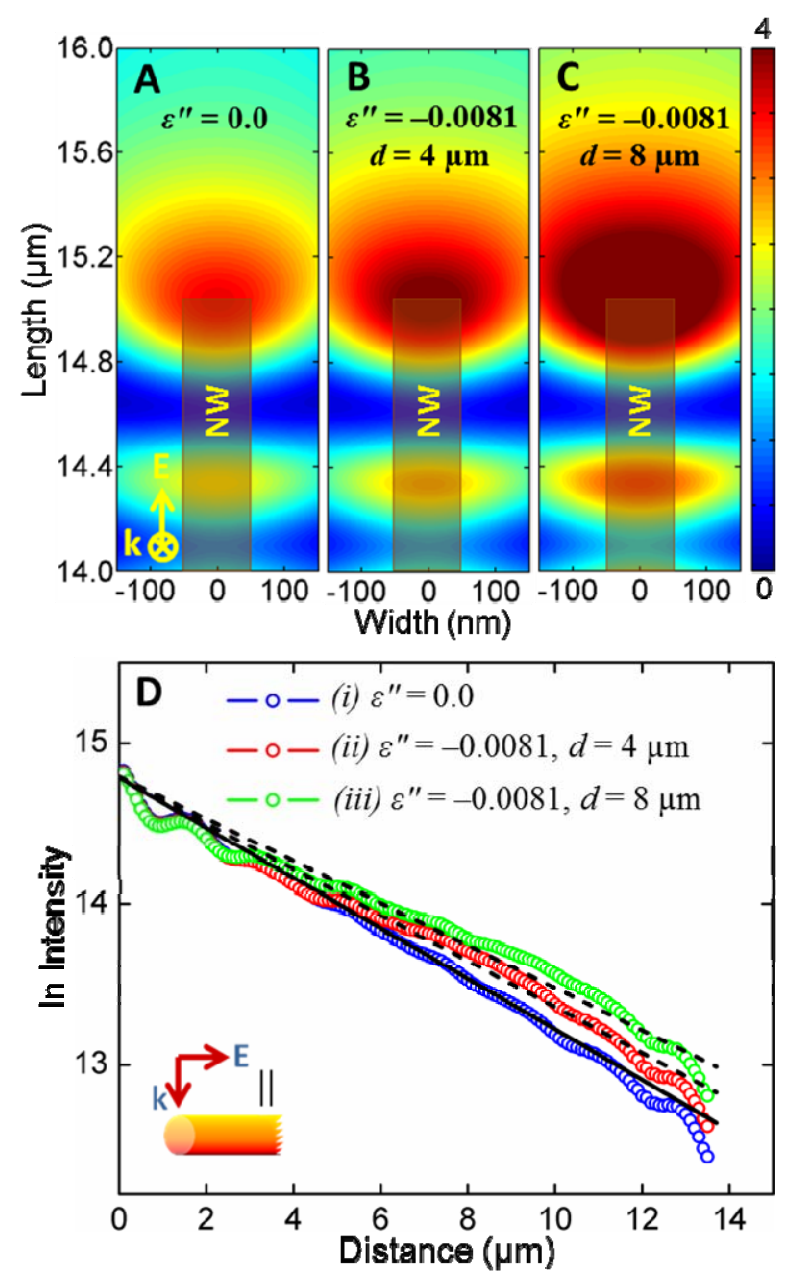

Figure 4. FDTD simulations of SPP gain. A $15 \mu \mathrm{m} \times 100 \mathrm{~nm} \mathrm{Ag} \mathrm{NW}$ was modeled on top of a dye gain medium with a permittivity having a real part of $\varepsilon^{\prime}=2.25$ and an adjustable imaginary part that reflects the amount of gain. (A) Top-views of the E-field intensity distribution around the output end of a Ag NW under $633 \mathrm{~nm}$ excitation at the opposite NW end (not shown) with laser polarization along the main NW axis when there is no gain, i.e. $\varepsilon^{\prime \prime}=0$. (B,C) Same E-field intensity distributions with a gain medium modeled by a value of $\varepsilon^{\prime \prime}=-0.0081$. The gain medium was circularly distributed around the center of the NW, with a diameter of $d=4 \mu \mathrm{m}(\mathrm{B})$ and $d=8$ $\mu \mathrm{m}(\mathrm{C})$. Larger E-field intensities at the NW end are clearly visible under gain conditions. E-field enhancement near the NW end around $14.4 \mu \mathrm{m}$ in $(\mathrm{A}-\mathrm{C})$ is due to the mode starting to leak from the NW at this position. The ratio of the maximum E-field intensities at the NW output ends, as obtained from the images, yields an enhancement factors of $f=1.21$ and $f=1.44$ for $d=4 \mu \mathrm{m}$ and $d=8 \mu \mathrm{m}$, respectively. (D) Decay of the E-field intensities along the main NW axis plotted on a natural logarithmic scale for the three cases: (i) no gain medium, (ii) $d=4 \mu \mathrm{m}$ gain region and (iii) $d=8 \mu \mathrm{m}$ gain region. While the solid black line corresponding to (i) is an actual fit to the data, yielding a propagation length of $L_{0}=6.4 \mu \mathrm{m}$, the dashed lines corresponding to (ii) and (iii) indicate the effective SPP decay for a uniformly distributed gain medium, assuming the same output enhancement factor (see text and Supporting Information for details). From both the Efield intensity distributions as well as the SPP decays, it is clear that increasing the size of the gain region leads to larger overall gain. Note that only light polarization parallel to the long NW axis was considered, because the $m=0$ mode, excited with longitudinal polarization, was $\sim 10^{3}$ times more intense than the $m=1$ mode, excited with transverse polarization (Figure S6). 
suggests that, in addition to stimulated emission of SPPs by the probe beam within the waveguide, conversion of SPPs to photons in the gain medium can lead to stimulated dye emission that then couples back into the waveguide and is detected at the output end.

Electromagnetic simulations using the finite difference time domain (FDTD) method support the experimental results (Figure 4). To model the optical pumping of the dye, the imaginary part of the gain medium, $\varepsilon^{\prime \prime}$, was chosen to be negative. The simulations assumed a circular gain region around the center of the NW with a diameter of $d=4 \mu \mathrm{m}$, equal to the size the $514 \mathrm{~nm}$ pump laser. A stronger electric field (E-field) intensity distribution at the $\mathrm{NW}$ end was observed with (i.e. $\varepsilon^{\prime \prime}=-0.0081$, Figure 4B) compared to without optical pumping (i.e. $\varepsilon^{\prime \prime}=0$, Figure 4A). From these E-field intensities, we calculated an output enhancement factor of $f=1.21$, which was matched to the experimental results shown in Figure 3 by adjusting the value of $\varepsilon^{\prime \prime}$. When the gain region was expanded to $d=8 \mu \mathrm{m}$, the E-field intensity at the NW end increased to give a larger output enhancement factor of $f=1.44$ (Figure 4C).

SPP gain was also analyzed from the E-field intensity decays along the NW without and with optical gain (Figure 4D). Without pumping, we obtain a SPP propagation length of $L_{0}=6.4 \mu \mathrm{m}$ (i). With pumping the E-field intensity could, however, not be fit to a single exponential decay, (ii) and (iii), because of the different slope in the region of localized optical gain. If the gain medium is instead distributed uniformly along the NW, this issue no longer exists, and we show in the Supporting Information (Figures S7 and S8) that for the same overall output enhancement factor the cases of a local vs. uniform gain regions are equivalent, allowing us to calculate an effective propagation length, $L^{*}$, under optical pumping even for a finite gain region. As indicated by the dotted 
lines for the data corresponding to (ii) and (iii) in Figure 4D, $L^{*}=7.0 \mu \mathrm{m}$ and $L^{*}=7.6$ $\mu \mathrm{m}$ for gain regions of $d=4 \mu \mathrm{m}$ and $d=8 \mu \mathrm{m}$, respectively.

Using the maximum enhancement factor of $f=1.50$ at $633 \mathrm{~nm}$ we determined a $14 \%$ reduction in SPP propagation losses, which we estimate to be $-0.85 \mathrm{~dB}_{\mu \mathrm{m}}^{-1}$ and $0.73 \mathrm{~dB}_{\mu \mathrm{m}}^{-1}$ without and with optical gain, respectively, by using an experimentally determined propagation length of $L_{0}=5.1 \mu \mathrm{m}$ for the unpumped waveguide (Figure S5). Furthermore, from the same enhancement factor of $f=1.50$ we compute an optical gain coefficient of $g=270 \mathrm{~cm}^{-1}$ using eqn. S7 (see Supporting Information for details). Further SPP loss compensation could be achieved by using a more stable chromophore or by pumping a larger area of the gain medium, with no dye molecules in the unpumped region to avoid absorptive losses.

In summary, we have successfully demonstrated optical gain by stimulated emission of SPPs at visible frequencies in single colloidal Ag NWs with a mode area of only $\lambda^{2} / 40$. The small mode area facilitates SPP gain at relatively low pump powers of the cw laser, ${ }^{20}$ as the highest pump power used here was at least an order of magnitude smaller than the time-integrated pump powers of the nanosecond pulsed lasers employed in previous studies. ${ }^{18-20}$ The achievement of $\mathrm{cw}$ gain for SPPs with subwavelength mode volumes is crucial for the future development of cw plasmonic amplifiers and subwavelength lasers.

\section{Acknowledgements}

This work was funded by the Robert A. Welch Foundation (C-1664, C-1222), ONR (N00014-10-1-0989), ARO (MURI W911NF-12-1-0407), and NSF (CHE-0955286). We 
would like to thank Jacob Khurgin, Christy Landes, Naomi Halas, and Mikael Käll for stimulating discussions.

\section{Author Contributions}

A.P. and S.L. conceived and designed the experiments, A.P. and W.S.C. implemented the experimental design. A.P. performed the experiments and analyzed the data. A.P. and S.L. prepared the manuscript. Y.R.Z. and P.N. carried out the simulations. Y.W. and Y.X. synthesized the samples. All authors contributed to writing the paper.

\section{Methods}

Sample Preparation. Pentahedrally twined Ag NWs stabilized by poly(vinyl pyrrolidone) (PVP) were synthesized by a wet chemical method described in the Supporting Information (Figure S1). The NWs were $15 \pm 1 \mu \mathrm{m}$ long with an average diameter of $100 \pm 20 \mathrm{~nm}$. Only NWs with lengths of $14-16 \mu \mathrm{m}$ were considered in the optical experiments. A $1 \mathrm{mM}$ solution of R640 dye (Exciton) with 4\% PMMA in anisole (Microchem) was spin-coated (Headway Research) onto clean glass coverslips (Fisher) at $1.5 \mathrm{~K} \mathrm{rpm}$ for 45 seconds. The combination of probe and pump wavelengths was chosen to be resonant with the R640 emission and absorption, respectively (Figure S2). NWs were then deposited onto these dye-doped polymer coated coverslips by dropcasting 200 $\mu \mathrm{L}$ of the appropriately diluted colloidal NW suspension. After 1 hour of drying under ambient conditions, the coverslips were washed with water and dried under a nitrogen flow. The average thickness of the dye-doped PMMA layer was determined by AFM 
(Veeco) to be around $250 \pm 20 \mathrm{~nm}$. Samples were studied optically within a week as they strongly degraded after this time period, likely due to oxidation of Ag.

SPP Gain Experiments. The detailed optical setup with excitation and detection geometries is described in the Supporting Information (Figure S3). The procedure for a typical SPP gain experiment was as follows: First, one end of an Ag NW was illuminated through a $100 \times$ microscope objective (oil, 1.3 N.A.) by a $633 \mathrm{~nm}$ probe laser with a low power of $12 \mathrm{~W} / \mathrm{cm}^{2}$, and an optical image was recorded using a CCD camera with an integration time set to typically 0.3 seconds. Next, the $633 \mathrm{~nm}$ beam was blocked, and a second image was recorded with a $514 \mathrm{~nm}$ pump laser beam $\left(100-800 \mathrm{~W} / \mathrm{cm}^{2}\right)$ focused in the middle of the NW, using a pair of steering mirrors to bring the $514 \mathrm{~nm}$ light to the desired spot. Next, both pump and probe lasers were turned on and a third image was recorded with the same integration time and same laser powers as before, although randomly the second and third steps were interchanged in order to exclude effects due to dye photobleaching (see also the Supporting Information). The pump beam was blocked by a 514 notch filter before the detector. To select only the probe wavelength in these image mode measurements, a $633 \mathrm{~nm}$ bandpass filter (Thorlabs) with a transmission window having a full width at half maximum (FWHM) of $1.0 \pm 0.2 \mathrm{~nm}$ was added to detection path. The intensity of the output signal was determined by taking a line section through the highest intensity pixel perpendicular to the NW in the measured CCD camera image. Both probe and pump beams were circularly polarized as control experiments revealed an insensitivity of the laser polarizations for our samples (Figure S4).

The experimental approach for spectra acquisition was similar as in image mode, except that an image of a NW excited with the $633 \mathrm{~nm}$ probe beam was recorded first, 
and then a 4 x 4 pixel region at the NW end was selected using a narrow slit opening and by vertical binning in order to monitor only light from the NW output. Experiments with a $50 \mu \mathrm{m}$ pinhole inserted into the microscope image plane to limit the integration area gave the same results. The CCD camera was then switched to spectrum mode and the spectrally resolved output signal was collected at the NW end only. In order to avoid errors associated with probe beam fluctuations, we carried out each gain experiment by recording 5-6 spectra for probe-only excitation before and after the measurements with pump-only and pump+probe excitations. An average probe beam spectrum was then constructed, like spectrum (i) shown in Figure 3A, and this spectrum was used to estimate the enhancement factor.

For the pump power dependence, the $f$ values according to eqn. 1 were determined at each power for $7 \mathrm{NWs}$ and these values were averaged to obtain a mean output enhancement factor. For a different pump power this procedure was repeated for a new set of NWs. Thus the error bar associated with each data point in Figure 2E indicates the standard deviation of the corresponding mean value at a certain pump power. This averaging procedure avoids issues associated with different input/output coupling efficiencies and variations in SPP propagation length because of the distribution of NW dimensions and tip geometries. Also, dye photobleaching restricts repeated gain measurements on the same NW especially at higher pump powers.

FDTD Simulations. Electromagnetic simulations using the FDTD method modeled an Ag NW with a length of $15 \mu \mathrm{m}$ and a diameter of $100 \mathrm{~nm}$, placed on a $250 \mathrm{~nm}$ thick dielectric layer with a real part of the medium permittivity of $\varepsilon^{\prime}=2.25$. Although the $\mathrm{Ag}$ NWs had a pentagonal cross sectional geometry (see inset to Figure S1A), the 
simulations assumed a cylindrical NW with a circular cross section, because, due to the small diameter of the Ag NWs, the corner modes hybridize into a symmetric fundamental SPP mode, which is similar to the $m=0$ mode supported by cylindrical NWs. ${ }^{30-32}$ A 633 nm Gaussian beam with a FWHM of $300 \mathrm{~nm}$ illuminated one end of the NW after first passing through the dielectric layer. For the complex dielectric function of $\mathrm{Ag}$, the tabulated values by Johnson and Christy were used..$^{33}$ The emitted energy flow from the output end of the NW was described by an E-field intensity distribution that was analyzed both in the sample plane as well as perpendicular to the main axis of the NW. To model the experimental gain conditions under $514 \mathrm{~nm}$ laser excitation, the imaginary part of the medium permittivity, $\varepsilon^{\prime \prime}$, was assumed to be negative and varied to yield a SPP gain as a function of pump irradiance consistent with experiment. For the localized gain simulations we assumed a circular gain region centered around the middle of the NW with an effective value of $\varepsilon^{\prime \prime}$ while the rest of the NW was surrounded by a dielectric with $\varepsilon^{\prime \prime}=0$. The values used for $\varepsilon^{\prime \prime}$ are consistent with previous studies on gain assisted plasmon propagation. ${ }^{17}$ Furthermore, $\varepsilon^{\prime \prime}$ was uniform in the z-direction away from the NW surface (with the NW in the $x y$ plane) and all experimental variations along this direction such as pump intensity, which decreases along the light propagation direction due to absorption, and emission quenching, which matters for molecules close to the NW surface, were averaged into an effective imaginary permittivity. Excellent agreement between experiment and simulation could be achieved in this way as further demonstrated in the Supporting Information (Figure S9). 


\section{References}

1. Schuller, J. A. et al. Plasmonics for extreme light concentration and manipulation. Nature Mater. 9, 193-204 (2010).

2. Gramotnev, D. K. \& Bozhevolnyi, S. I. Plasmonics beyond the diffraction limit. Nature Photon. 4, 83-91 (2010).

3. Barnes, W. L., Dereux, A. \& Ebbesen, T. W. Surface plasmon subwavelength optics. Nature 424, 824-830 (2003).

4. Lin, J. et al. Polarization-controlled tunable directional coupling of surface plasmon polaritons. Science 340, 331-334 (2013).

5. Esteban, R., Borisov, A. G., Nordlander, P. \& Aizpurua, J. Bridging quantum and classical plasmonics with a quantum-corrected model. Nat. Commun. 3, 825 (2012).

6. Anderson, L. J. E., Zhen, Y.-R., Payne, C. M., Nordlander, P. \& Hafner, J. H. Gold nanobelts as high confinement plasmonic waveguides. Nano Lett. 13, 62566261 (2013).

7. Lee, S. J. \& Moskovits, M. Remote sensing by plasmonic transport. J. Am. Chem. Soc. 134, 11384-11387 (2012).

8. Lal, S., Hafner, J. H., Halas, N. J., Link, S. \& Nordlander, P. Noble metal nanowires: From plasmon waveguides to passive and active devices. Acc. Chem. Res. 45, 1887-1895 (2012).

9. Kusar, P., Gruber, C., Hohenau, A. \& Krenn, J. R. Measurement and reduction of damping in plasmonic nanowires. Nano Lett. 12, 661-665 (2012).

10. Ditlbacher, H. et al. Silver nanowires as surface plasmon resonators. Phys. Rev. Lett. 95, 257403 (2005).

11. Allione, M., Temnov, V. V., Fedutik, Y., Woggon, U. \& Artemyev, M. V. Surface plasmon mediated interference phenomena in low-q silver nanowire cavities. Nano Lett. 8, 31-35 (2008).

12. Fang, Y. R. et al. Branched silver nanowires as controllable plasmon routers. Nano Lett. 10, 1950-1954 (2010).

13. Dorfmuller, J. et al. Fabry-perot resonances in one-dimensional plasmonic nanostructures. Nano Lett. 9, 2372-2377 (2009).

14. Raether, H. Surface plasmons on smooth and rough surfaces and on gratings. (Springer, 1988).

15. Berini, P. \& De Leon, I. Surface plasmon-polariton amplifiers and lasers. Nature Photon. 6, 16-24 (2012).

16. Noginov, M. A. et al. Stimulated emission of surface plasmon polaritons. Phys. Rev. Lett. 101, 226806 (2008).

17. Grandidier, J. et al. Gain-assisted propagation in a plasmonic waveguide at telecom wavelength. Nano Lett. 9, 2935-2939 (2009).

18. Gather, M. C., Meerholz, K., Danz, N. \& Leosson, K. Net optical gain in a plasmonic waveguide embedded in a fluorescent polymer. Nature Photon. 4, 457$461(2010)$.

19. De Leon, I. \& Berini, P. Amplification of long-range surface plasmons by a dipolar gain medium. Nature Photon. 4, 382-387 (2010). 
20. Kéna-Cohen, S., Stavrinou, P. N., Bradley, D. D. C. \& Maier, S. A. Confined surface plasmon-polariton amplifiers. Nano Lett. 13, 1323-1329 (2013).

21. Suh, J. Y. et al. Plasmonic bowtie nanolaser arrays. Nano Lett. 12, 5769-5774 (2012).

22. Noginov, M. A. et al. Demonstration of a spaser-based nanolaser. Nature 460, 1110-1112 (2009).

23. Seidel, J., Grafström, S. \& Eng, L. Stimulated emission of surface plasmons at the interface between a silver film and an optically pumped dye solution. Phys. Rev. Lett. 94, 177401 (2005).

24. Ambati, M. et al. Observation of stimulated emission of surface plasmon polaritons. Nano Lett. 8, 3998-4001 (2008).

25. Noginov, M. A. et al. Compensation of loss in propagating surface plasmon polariton by gain in adjacent dielectric medium. Opt. Express 16, 1385-1392 (2008).

26. Solis, D. et al. Bleach-imaged plasmon propagation (blipp) in single gold nanowires. Nano Lett. 10, 3482-3485 (2010).

27. Wild, B. et al. Propagation lengths and group velocities of plasmons in chemically synthesized gold and silver nanowires. ACS Nano 6, 472-482 (2012).

28. Paul, A. et al. Identification of higher order long-propagation-length surface plasmon polariton modes in chemically prepared gold nanowires. ACS Nano 6 , 8105-8113 (2012).

29. Wei, H. et al. Quantum dot-based local field imaging reveals plasmon-based interferometric logic in silver nanowire networks. Nano Lett. 11, 471-475 (2011).

30. Song, M. et al. Imaging symmetry-selected corner plasmon modes in pentatwinned crystalline ag nanowires. ACS Nano 5, 5874-5880 (2011).

31. Zhang, S. \& Xu, H. Optimizing substrate-mediated plasmon coupling toward high-performance plasmonic nanowire waveguides. ACS Nano 6, 8128-8135 (2012).

32. Nauert, S. et al. Influence of cross sectional geometry on surface plasmon polariton propagation in gold nanowires. ACS Nano, In press (2013).

33. Johnson, P. B. \& Christy, R. W. Optical constants of the noble metals. Phys. Rev. B 6, 4370-4379 (1972). 